\title{
Alterstice
}

Revue internationale de la recherche interculturelle

International Journal of Intercultural Research

Revista International de la Investigacion Intercultural

\section{Éveil aux langues et évolution des représentations d'élèves plurilingues sur leur répertoire linguistique}

\section{Marie-Paule Lory et Françoise Armand}

Volume 6, numéro 1, 2016

Prendre en compte la diversité à l'école

URI : https://id.erudit.org/iderudit/1038276ar

DOI : https://doi.org/10.7202/1038276ar

Aller au sommaire du numéro

Éditeur(s)

Alterstice

ISSN

1923-919X (numérique)

Découvrir la revue

Citer cet article

Lory, M.-P. \& Armand, F. (2016). Éveil aux langues et évolution des représentations d'élèves plurilingues sur leur répertoire linguistique. Alterstice, 6(1), 27-38. https://doi.org/10.7202/1038276ar

\section{Résumé de l'article}

Même si les bénéfices d'une éducation plurilingue sont largement documentés, la diversité linguistique et culturelle ne fait pas toujours l'objet d'une valorisation par le système scolaire. À Montréal notamment, où la diversité linguistique est prégnante, les élèves plurilingues sont susceptibles de vivre, à des degrés divers, des rapports de force entre les langues qui les entourent. Il semble que le système éducatif québécois promeuve aujourd'hui encore prioritairement, voire uniquement, la langue et la culture de la majorité. C'est pour cette raison qu'au moyen de l'implantation d'activités pédagogiques d'éveil aux langues nous avons étudié, sur deux ans, les représentations de cinq élèves plurilingues scolarisés en fin de cycle du primaire sur les langues de leur répertoire linguistique. Plus particulièrement, les résultats de la recherche qualitative présentés dans cet article s'articulent autour de trois dimensions qui ont participé à la dynamisation des répertoires linguistiques des participants à la recherche : le temps, le contexte et les interlocuteurs. 


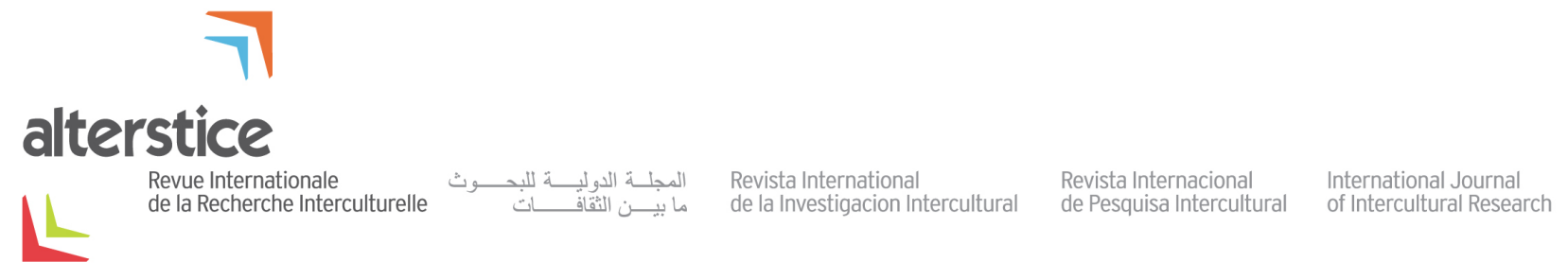

ARTICLE THÉMATIQUE

\title{
Éveil aux langues et évolution des représentations d'élèves plurilingues sur leur répertoire linguistique
}

\author{
Marie-Paule Lory ${ }^{1}$ et Françoise Armand ${ }^{2}$
}

\section{Résumé}

Même si les bénéfices d'une éducation plurilingue sont largement documentés, la diversité linguistique et culturelle ne fait pas toujours l'objet d'une valorisation par le système scolaire. À Montréal notamment, où la diversité linguistique est prégnante, les élèves plurilingues sont susceptibles de vivre, à des degrés divers, des rapports de force entre les langues qui les entourent. II semble que le système éducatif québécois promeuve aujourd'hui encore prioritairement, voire uniquement, la langue et la culture de la majorité. C'est pour cette raison qu'au moyen de l'implantation d'activités pédagogiques d'éveil aux langues nous avons étudié, sur deux ans, les représentations de cinq élèves plurilingues scolarisés en fin de cycle du primaire sur les langues de leur répertoire linguistique. Plus particulièrement, les résultats de la recherche qualitative présentés dans cet article s'articulent autour de trois dimensions qui ont participé à la dynamisation des répertoires linguistiques des participants à la recherche : le temps, le contexte et les interlocuteurs.

\section{Rattachement des auteures}

${ }^{1}$ Université de Toronto, Toronto, Canada ; ${ }^{2}$ Université de Montréal, Montréal, Canada

\section{Correspondance}

mariepaule.lory@utoronto.ca

\section{Mots clés}

éveil aux langues, représentations sur les langues, plurilinguisme et école, répertoire linguistique.

\section{Pour citer cet article}

Lory, M.-P. et Armand, F. (2016). Éveil aux langues et évolution des représentations d'élèves plurilingues sur leur répertoire linguistique. Alterstice, 6(1), 27-38. 


\section{Introduction}

Si 7097 langues ont été recensées comme étant parlées dans le monde (Ethnologue, 2015), seulement 50 d’entre elles sont parlées par plus de la moitié de la population (Moseley, 2010 ; UNESCO, 2003). À l'école, la diversité linguistique est quant à elle encore moins représentée (Cummins, 2014), et ce, même si depuis des décennies, de nombreux chercheurs ont souligné l'inadéquation des modèles pédagogiques actuels quant à la prise en compte de la diversité culturelle et linguistique en contexte scolaire pluriel (Armand, Dagenais et Nicollin, 2008; Cummins, 2001, 2007 et 2014 ; Cummins et Early, 2011 ; Garcia et Kleifgen, 2010 ; Hélot, 2007 ; Hélot et Ò Laoire, 2011 ; Lüdi et Py, 2009 ; Moore, 2006 ; Prasad, 2015). D’ailleurs, ces chercheurs adjurent les milieux scolaires de laisser une place aux langues de l'immigration dans les classes et de permettre, au moyen d'approches pédagogiques appropriées, de valoriser le répertoire linguistique et culturel des élèves immigrants ou issus de l'immigration.

C'est dans cet esprit que, pendant deux ans, dans deux classes du primaire de fin de troisième cycle (enfants de 10 à 12 ans) à Montréal, un projet d'éveil aux langues (Élodil) a été implanté auprès d'élèves plurilingues immigrants et issus de l'immigration. Précisons que, en raison de sa politique d'immigration, le Québec est la troisième province canadienne à accueillir le plus d'immigrants. La majorité d'entre eux viennent s'installer sur l'île de Montréal (Statistique Canada, 2011). Les écoles montréalaises sont donc le miroir d’une diversité linguistique et culturelle croissante. Néanmoins, la politique scolaire ne laisse que peu (voire pas) de place aux langues parlées par les élèves au sein de la classe et favorise clairement la langue officielle du Québec, le français (Armand, Dagenais et Nicollin, 2008). C'est dans ce contexte que nous avons participé à l'implantation du projet Élodil qui, au moyen d'activités pédagogiques novatrices, souhaite faire de la salle de classe un espace de complicité linguistique où la langue de scolarisation, les langues des élèves, les langues du quartier et plus largement les langues du monde deviennent des sources de savoir, d'apprentissage et de discussions (Élodil, 2010). L'implantation de ces activités a fait, entre autres, l'objet d'une recherche doctorale qui a permis de documenter l'évolution des représentations de cinq élèves du primaire sur les langues (Lory, 2015). Comme le soulignent Castellotti et Moore (2002), documenter les représentations sur les langues permet de rendre compte de façon holistique des ressources mobilisées dans l'apprentissage des langues. De plus, cela permet de tenir compte des dimensions affective et identitaire en jeu dans le développement langagier.

Dans le cadre de cet article, nous nous concentrons sur ce qui représente un obstacle ou un soutien à l'expression de représentations positives sur les langues du répertoire linguistique de ces élèves. Nous commençons par définir les termes clés et les théories au fondement de cette recherche, puis nous exposons la méthodologie adoptée pour finalement présenter, dans la section des résultats, trois dimensions présentes dans la dynamisation des répertoires linguistiques des participants (le temps, le contexte et les interlocuteurs).

\section{Cadre conceptuel}

Afin de mieux appréhender les concepts clés et théories en lien avec cette recherche, nous proposons une articulation en en quatre sous-sections.

\section{Prendre en compte la diversité linguistique en contexte scolaire}

Genesee, Paradis et Crago (2004) ont souligné que certaines croyances ou représentations sociales sur l'apprentissage des langues incitent de nombreux parents et enseignants, en contexte d'immigration, à favoriser la langue de la société d'accueil. Dès lors, dans les salles de classe, les langues parlées par les élèves sont trop souvent passées sous silence et l'accent est clairement mis sur la ou les seules langues d'enseignement (Cummins, 2007, 2014). Toutefois, les recherches se multiplient pour démontrer que renier l'héritage linguistique et culturel des élèves immigrants ou issus de l'immigration peut avoir des effets néfastes sur les élèves, et ce, à plusieurs égards. Tout d'abord, sur le plan socioaffectif, Armand, Dagenais et Nicollin (2008), s'appuyant sur de nombreux auteurs, soulignent que la non-reconnaissance des langues parlées par les élèves, quand elles sont autres que la langue de scolarisation, peut placer certains élèves en "insécurité linguistique » et est susceptible de les amener à développer « un sentiment de discrimination, une baisse de l'estime de soi, ainsi que par des difficultés à transférer des acquis cognitifs et langagiers d'une langue à l'autre »(p. 47). Ensuite, sur les plans cognitif et langagier, des 
chercheurs tels que Lambert, avec sa théorie sur "les situations de bilinguisme additif/ soustractif » (dans Hamers, 2005, p. 280), ou Cummins, avec ses théories sur «l'hypothèse d'interdépendance des langues, la compétence commune sous-jacente et le niveau seuil minimal de développement linguistique » (2007, p. 173), ont largement pu démontrer les bénéfices de la prise en compte des langues d'origine dans l'apprentissage de la langue d'accueil. Ainsi, une fois atteint un certain seuil de compétence (Cummins, 2007), le développement langagier dans la ou les langue(s) maternelle(s) peut se poursuivre en parallèle de la langue d'accueil sans que ce développement ne représente un frein dans les nouveaux apprentissages. Hamers (2005) mentionne d'ailleurs que, pour que l'apprenant puisse pleinement bénéficier de cette éducation bilingue, il faut que, d'une part, il ait appris à manipuler le langage pour des activités cognitives complexes qui incluent souvent des activités métalinguistiques et que, d'autre part, la ou les langue(s) maternelle(s) de l'apprenant et la langue de la société d'accueil soient valorisées dans l'entourage de l'apprenant.

Ces différentes recherches s'accordent donc sur le fait que si le système scolaire n'est pas en mesure de prendre en compte le répertoire linguistique pluriel de ses élèves, on peut supposer que certains d'entre eux ne se sentiront pas acceptés dans leur pluralité et, surtout, ne s'autoriseront pas à réaliser des transferts langagiers pouvant favoriser leur apprentissage de la langue cible. Également, il paraît important de considérer les conditions sociolinguistiques dans lesquelles se déroulent le maintien et l'apprentissage dit complémentaire (et non en opposition) de deux langues (ou plus) chez les apprenants (Hamers, 2005). L'approche d'éveil aux langues, telle que nous la définissons dans la section suivante avec le projet Élodil, représente à cet égard une voie à investir, dans la mesure où elle participe au développement de représentations positives sur les langues et offre ainsi un espace pour développer des apprentissages langagiers de façon harmonieuse.

\section{L'éveil aux langues et le projet Élodil}

L'approche d'éveil aux langues est une approche pédagogique dite plurielle (Candelier, 2008) qui se situe à l'opposé de nombreuses approches coercitives traditionnellement mises en place dans les milieux scolaires, où seule la langue de la majorité prévaut. Elle engage une révision des rapports de pouvoir entre les langues instaurées par la société dominante et la mise en place d'une collaboration au sein de la classe sur laquelle se fonde l'engagement dans des activités pédagogiques. Les langues, dont certaines sont inconnues des élèves tandis que d'autres sont les langues d'un ou de plusieurs élèves de la classe, y sont un objet d'apprentissage, de discussion et de réflexion. Cet aller-retour entre les langues du monde et les langues de la classe permet de «montrer que la situation de multilinguisme est une situation très banale, qu'elle est la normalité même de l'humain, et de mettre les langues habituellement dévalorisées dans le même paradigme que de grandes langues internationales » (Candelier, 2006, p. 20). Dès lors, les activités d'éveil aux langues peuvent permettre de développer chez les élèves des représentations positives face à la diversité linguistique et culturelle, accroître leur motivation à l'apprentissage des langues et construire des aptitudes favorables au processus d'acquisitionapprentissage des langues (Dompmartin-Normand, 2003). En ce sens, cette approche est susceptible de toucher les dimensions cognitive, affective, sociale, identitaire et interculturelle.

Au Canada, l'approche d'éveil aux langues est notamment connue grâce au projet Élodil (Armand, 2012 ; Armand, Dagenais et Nicollin, 2008 ; Élodil, 2010). Les activités pédagogiques développées dans le cadre de ce projet ont pour divers buts :

- permettre aux élèves de développer des attitudes positives face à la diversité linguistique et culturelle ;

- faciliter la reconnaissance et la légitimation des langues d'origine des enfants immigrants allophones en contexte pluriethnique ;

- développer des connaissances et des capacités de réflexion sur les langues (capacités métalinguistiques) ;

- favoriser la réflexion sur le rôle social et identitaire du français langue commune en milieu québécois francophone. 
Les activités peuvent prendre plusieurs formes :

- la découverte de corpus oraux, où par exemple les élèves écoutent des pairs dire bonjour et se présenter dans plusieurs langues (module intitulé Les Bonjours) ou écoutent-des bulletins météos en créole haïtien, espagnol, malgache et inuktitut (module intitulé Parler de la pluie et du beau temps) ;

- des activités de comparaisons de langues où, à titre d'illustration, les élèves sont amenés à découvrir et travailler autour du concept de familles de langues (Les langues en contact) ;

- des activités réflexives autour de leur répertoire plurilingue au moyen de biographies langagières (Dis-moi ta/tes langues et je te dirai qui tu es) et de leur environnement plurilingue (À la découverte de mon quartier) ;

- des activités de découverte et d'écriture de proverbes d'ici et d'ailleurs (Les proverbes à travers le monde).

Dès lors, le projet Élodil offre un espace propice à une réflexion sur les langues, et plus particulièrement sur les langues du répertoire linguistique des élèves.

\section{Les répertoires linguistiques des élèves et la notion de compétences partielles}

Hélot (2007) mentionne qu'un répertoire linguistique se compose de «l'ensemble des variétés linguistiques que possède un individu, posé comme acteur social, membre d’un ou plusieurs groupes et engagé dans des interactions collectives, et dont il peut jouer selon les situations et les besoins de communications » (p. 52). Moore (2006) précise qu'un individu peut avoir des compétences plus ou moins partielles dans l'une ou l'autre de ses langues (ou variétés linguistiques) et être en mesure de les utiliser, ou de vouloir les utiliser ou non, selon les contextes. Ainsi, un individu bilingue "n'est pas définissable comme résultant de l'addition de deux répertoires monolingues" (Deprez, 1994, p. 26), mais il dispose d'une compétence plus large: une compétence dite plurilingue et pluriculturelle qui considère à la fois les aspects langagier et identitaire (Moore, 2006). En définissant de la sorte un individu bi-plurilingue, les modèles traditionnels axés uniquement sur le concept de compétence parfaite s'effacent au profit d'une vision plus dynamique et multidimensionnelle du bi-plurilinguisme dans laquelle l'aspect identitaire (Cummins et Early, 2011; Norton et Toohey, 2011;) et l'aspect émotionnel (Puozzo Capron et Picardo, 2013) occupent une place à part entière.

Le répertoire plurilingue et pluriculturel des élèves est alors perçu comme un atout (Bono et Stratilaki, 2009) dans le processus d'apprentissage. Toutefois, pour que les apprenants considèrent leur répertoire plurilingue comme un atout, il semble nécessaire qu'ils développent des représentations positives sur ce répertoire, en s'écartant des représentations sociales traditionnelles qui considèrent la compétence parfaite dans une langue comme seule compétence louable.

\section{Les représentations sur les langues}

La notion de représentations sociales constitue un objet d'étude prisé dans de nombreux domaines des sciences humaines comme l'anthropologie, la psychologie, l'économie, la sociologie ou encore l'éducation. Elle prend ses racines en psychologie sociale avec Moscovici et Jodelet (voir Jodelet, 1989) qui la définissent comme une façon de penser la réalité construite en interaction avec un groupe de référence. Plus spécifiquement adaptée à la didactique des langues, on retrouve la notion de représentations sur les langues. Ces dernières sont alors définies comme l'ensemble des images qu'un individu se construit à propos des langues (Lory, 2015). La pertinence de l'étude des représentations en contexte scolaire est soulignée par Castellotti et Moore (2002), qui mentionnent qu'en raison de "l'hétérogénéité de la notion» (p.9), elle permet aux chercheurs de rendre compte de la multiplicité des sources mobilisées dans l'apprentissage et l'enseignement des langues.

C'est donc dans ce cadre conceptuel que s'ancre la recherche présentée ici. La section suivante offre un aperçu de la démarche méthodologique adoptée, et elle sera suivie de la présentation des résultats. 


\section{Méthodologie}

Dörnyei (2007) souligne la pertinence de la mise en place de recherches qualitatives dans les études longitudinales, car elles nous permettent d'explorer les patterns et les changements qui se produisent chez les participants étudiés. C'est dans cette lignée que nous avons adopté un positionnement descriptif et interprétatif afin de documenter de façon nuancée l'évolution des représentations de cinq élèves sur les langues de leur répertoire linguistique sur une période de deux ans, dans le cadre de l'implantation d'un projet d'éveil aux langues. En ce sens, notre recherche s'apparente à une étude multicas (Stake, 2006) dans laquelle chaque élève représente un cas. Cette posture méthodologique nous permet de rendre compte de la complexité des représentations sur les langues, des différents facteurs impliqués et des phénomènes participant à la construction et l'évolution des représentations sur les langues de nos participants tout en respectant la singularité de chacun des cas à l'étude.

Il convient de préciser que les résultats présentés sont extraits des résultats d'une thèse de doctorat (Lory, 2015) qui s'est inscrite dans le cadre d'une vaste recherche à visée comparative entre Montréal et Vancouver ${ }^{1}$.

\section{Le terrain}

La recherche a pris place dans une école primaire publique montréalaise pluriethnique et plurilingue où des activités d'éveil aux langues ont été implantées dans deux classes de fin de cycle du primaire.

Précisons qu'entre 2006 et 2008, lors de la période de la collecte de données, dans les écoles publiques montréalaises qui ont le français comme langue d'enseignement, presque $50 \%$ des élèves déclaraient que le français n'était pas leur langue maternelle. De plus, le quartier dans lequel a eu lieu la recherche est l'un des quartiers les plus pluriethniques de Montréal et a également un indice de défavorisation plutôt élevé (CGTSIM, 2013). II convient d'ailleurs de noter que, dans les deux classes dans lesquelles nous sommes intervenues, aucun des élèves n'est monolingue.

\section{Les participants}

Le projet Élodil a été mis en place dans deux classes de $5^{\mathrm{e}}$ année pour la première année de l'intervention et nous avons suivi ces mêmes élèves pendant la seconde année de l'intervention, c'est-à-dire durant leur $6^{\mathrm{e}}$ année. Tous les élèves de ces classes ont pu participer au projet. Néanmoins, plusieurs d'entre eux ont été spécifiquement ciblés à des fins de recherche. De façon pratique, les « élèves Élodil », comme eux-mêmes aimaient s'appeler,

- étaient enregistrés pendant les activités de classe (supports audio ou vidéo),

- étaient l'objet de prises de notes réalisées par la ou les chercheure(s) présente(s),

- ont participé à trois entrevues semi-dirigées dans le but spécifique de documenter leurs représentations sur les langues.

Le tableau 1 offre une description des cinq participants à cette recherche.

\footnotetext{
${ }^{1}$ Dagenais, Lamarre, Moore et Sabatier, 2005-2008, Éveil aux langues et à la diversité linguistique dans deux métropoles canadiennes (Montréal, Vancouver), subvention CRSH. L'implantation du projet à Montréal a été dirigée par F. Armand et coordonnée par M.-P. Lory.
} 
Tableau 1 Données sociodémographiques des participants

\begin{tabular}{|c|c|c|c|}
\hline Prénoms* & $\begin{array}{c}\text { Année et lieu de } \\
\text { naissance }\end{array}$ & Pays d'origine des parents & $\begin{array}{c}\text { Langues entendues ou } \\
\text { parlées à la maison }\end{array}$ \\
\hline Laura & 1996, Montréal & Bolivie & Espagnol \\
\hline Shawn & 1996, Montréal & $\begin{array}{l}\text { Mère : Grenade } \\
\text { Père : Seychelles }\end{array}$ & Français, anglais, créole \\
\hline Oliver & 1995, Montréal & $\begin{array}{l}\text { Mère : Haïti } \\
\text { Père : Seychelles }\end{array}$ & Français, anglais, créole \\
\hline Savana & 1996, Bangladesh & Bangladesh & Français, anglais, bengali \\
\hline Evan & 1996, Montréal & $\begin{array}{l}\text { Mère : Cambodge } \\
\text { Père : Chine }\end{array}$ & Mandarin, français, anglais \\
\hline
\end{tabular}

* Pour des raisons de confidentialité, des prénoms fictifs ont été donnés aux participants.

\section{Description de l'intervention Élodil sur deux ans : activités en classe et entrevues de groupe}

Les activités d'éveil aux langues implantées en salle de classe sont issues du projet Élodil (Élodil, 2010) et se divisent en ce que nous appelons des modules. Chaque module traite d'une thématique différente. Par exemple, le module 1 intitulé À la découverte de notre quartier aborde les langues présentes dans le quartier où se situe l'école des élèves participant à la recherche et il est constitué de sept rencontres. Chaque module est traité en une ou plusieurs rencontres, d'une heure environ (entre 55 et 75 minutes) et, au total, 32 rencontres réparties en sept modules ont été implantées durant les deux ans d'intervention. Précisons que la majorité des activités a été prise en charge par les enseignants titulaires des classes impliquées dans la recherche et que ces enseignants ont reçu une formation à cet effet.

Les élèves ciblés dans cette recherche ont participé à trois entrevues de groupe semi-dirigées. La première a eu lieu avant l'implantation du projet d'éveil aux langues, la deuxième à la fin de la première année d'intervention et la troisième, à la fin de la seconde année d'intervention. L'entrevue de groupe a été privilégiée en raison de son caractère social (Lüdi et Py, 2002). Les élèves participant aux entrevues étaient donc en situation d'interaction, ce qui favorisait l'émergence de représentations sur les langues. Ces entrevues de groupe étaient semi-dirigées afin d'offrir une certaine flexibilité dans les échanges. Le guide d'entrevue exploité durant chacune des entrevues s'articulait autour de quatre grandes thématiques:

- les représentations sur la fonction de la langue,

- les représentations (attraction / rejet) de certaines langues,

- les représentations sur le bilinguisme / multilinguisme,

- les représentations sur les langues du quartier.

\section{Les types de données et leur traitement}

Plusieurs types de données ont été collectées. Chaque séance d'activités ainsi que les entrevues ont fait l'objet d'enregistrements à la fois vidéo et audio. Ces enregistrements ont été retranscrits, constituant une première source de données. Divers productions d'élèves ont également été analysées. Ces productions sont variables et prennent diverses formes, par exemple :

- un pétale de fleur sur lequel les élèves écrivent les langues qu'ils parlent (module La fleur des langues),

- des réponses à un questionnaire sur les langues qu'ils aimeraient découvrir (module Les contes),

- une biographie langagière présentée devant la classe (module Dis-moi ta/tes langues et je te dirais qui tu es !).

De plus, des notes de terrain prises pendant les rencontres Élodil ainsi que durant les entrevues sont venues alimenter notre analyse. 
En ce qui a trait au traitement des données, nous avons adopté une logique d'analyse inductive et récursive propre à l'approche qualitative (Paillé, 1996). Nous avons procédé à une analyse de contenu (Krippendorff, 2013) afin de rendre compte, le plus objectivement possible, du discours des participants : à partir d'une grille d'analyse, nous avons codé les informations recueillies au moyen du logiciel QDA Miner pour ensuite procéder à leur analyse. Cette dernière s'est inspirée des analyses de discours utilisées dans le domaine de la communication (Maingueneau, 2007).

\section{Résultats}

Garcia (2009) et Moore et Castellotti (2008) soulignent que les répertoires linguistiques des élèves immigrants et issus de l'immigration sont marqués par une forme de dynamisme. L'analyse qualitative des données recueillies durant les deux ans d'implantation du projet Élodil illustre clairement ce dynamisme. Plus particulièrement, en nous appuyant sur les représentations des cinq participants à la recherche sur les langues de leur répertoire, Evan, Laura, Oliver, Savana et Shawn, nous avons pu faire émerger trois dimensions qui contribuent au dynamisme des répertoires linguistiques de ces élèves: le temps, le contexte et les interlocuteurs. Nous présentons donc une analyse des discours recueillis pendant l'intervention articulée autour de ces trois dimensions.

\section{Dynamisme des répertoires linguistiques des participants dans le temps}

L'analyse des données montre que le répertoire linguistique des participants s'inscrit à la fois dans le passé, dans le présent et dans le futur. Quatre élèves sur cinq modifient au fil des interventions leurs déclarations quant aux langues en présence dans leur répertoire linguistique.

Par exemple, lors de la première entrevue, soit avant toute intervention pédagogique, Savana déclare comme langues parlées le français et le bengali. Elle précise que le bengali est sa langue maternelle et la langue qu'elle parle avec ses parents et sa fratrie (entrevue 1). Après neuf rencontres Élodil, elle intègre l'anglais à son répertoire reconnaissant qu'elle apprend cette langue à l'école. Lors de la deuxième entrevue, qui conclue la première année d'intervention, Savana interroge l'enquêtrice sur la place qu'elle peut attribuer à l'arabe dans la mesure où elle suit un enseignement coranique dans cette langue et qu'elle peut lire dans cette langue, mais pas parler. Ces premières évolutions font référence à des langues qui, dans le présent, font partie de l'environnement linguistique de l'élève. Il est alors intéressant de noter que, lors de la seconde année du projet, c'est l'ourdou qui prend une place à part entière au sein de son répertoire et elle indique, suite à un module portant sur l'écriture de sa biographie langagière (module 6, Dis moi ta/tes langues et je te dirai qui tu es !) :

J'ai eu, en tout, quatre professeurs : I'un me parlait en bengali et les trois autres de temps en temps en anglais et d'autres temps en ourdou. C'est de cette façon que j'ai appris l'ourdou. (Savana, module 6)

C'est donc après 26 séances que Savana fait explicitement référence à ses apprentissages scolaires antérieurs, dans son pays d'origine. Manifestement, c'est ce regard vers le passé qui lui permet d'ouvrir la porte à l'intégration de l'ourdou au sein de son répertoire linguistique, puisque l'ourdou sera désormais toujours cité comme langue de son répertoire. Toujours lors de la présentation de sa biographie langagière, Savana se projette cette fois dans le futur en indiquant :

Je n'ai surtout pas envie d'avoir un métier que je n'aime pas, je veux que toutes les portes du monde s'ouvrent pour moi puisque plus que tu parles de langues, plus tu as des chances de pratiquer un métier intéressant et ensuite avec mes économies, je partirai faire le tour du monde. Mais bien sûr, l'anglais et le français ne suffisent pas pour faire le tour du monde et puis je sais qu'en vieillissant j'apprendrai d'autres langues. (Savana, module 6)

Nous avons ici illustré comment le dynamisme du répertoire de Savana s'inscrit dans le temps. Des évolutions similaires ont été repérées autour des répertoires linguistiques de Laura, Oliver et Shawn. Dans cette lignée, il apparaît pertinent de préciser que la légitimation plus ou moins rapide de certaines langues au sein du répertoire des élèves est en partie liée au fait que les élèves reçoivent (ou ont reçu) ou non un enseignement explicite dans ces langues. C'est le cas par exemple de l'anglais, qui est enseigné à l'école et qui, pour Savana comme pour Laura, était passé sous silence lors de la première entrevue. Après neuf séances d'éveil aux langues, et justifié par 
l'enseignement reçu en anglais dans le système scolaire québécois, ces deux élèves intègrent l'anglais comme langue de leur répertoire.

Quand les langues ne font pas l'objet d'un enseignement formel, leur intégration dans le répertoire linguistique des élèves doit davantage être négociée. Le sentiment d'insécurité linguistique en lien avec la vision d'une compétence parfaite dans l'une ou l'autre des langues est au cœur de cette négociation pour tous les élèves participant à la recherche. Si ces représentations sociales traditionnelles de ce qu'est être un locuteur plurilingue (Baker, 2011) influencent le discours initial des élèves, au fil des interventions, ils jouent de stratégies afin de légitimer l'intégration de l'une ou l'autre des langues dans leur répertoire :

- ils apportent un jugement de valeur sur leurs compétences, par exemple Savana avec l'anglais, qui dit qu'elle parle « un peu l’anglais » et qu'elle sait « se débrouiller » dans cette langue (entrevue 3),

- ils spécifient leur domaine de compétence : Shawn qui indique qu'il « comprend » le créole (module 6),

- ils qualifient, étiquettent leurs langues : Laura intègre l'aymara et le quechua au sein de son répertoire en tant que langues d'origine « non parlées » (module 6).

Grosjean (1982) souligne que les représentations traditionnelles du bilinguisme construites sur une perspective " monolingue » des compétences langagières est à l'origine des attitudes négatives vis-à-vis des compétences des bilingues. Ces représentations sont largement véhiculées par la société et sont susceptibles de positionner les élèves en insécurité linguistique. Cette insécurité linguistique est une composante intégrante du discours chez chacun des participants à la recherche. Néanmoins, l'implantation des activités Élodil permet aux élèves de revisiter ces représentations, et le sentiment d'insécurité linguistique initialement exprimé s'estompe au fil du temps chez quatre participants sur cinq.

Le contexte : I'intégration d'une norme scolaire monolingue qui influence les répertoires linguistiques des participants

Blommaert, Collins et Slembrouck (2005) soutiennent que le plurilinguisme n'est pas simplement lié aux compétences individuelles dans l'une ou l'autre des langues, mais qu'il est également et surtout situé dans un contexte sociohistorique singulier et marqué par des politiques linguistiques spécifiques. Le contexte scolaire québécois est de toute évidence à considérer dans la dynamique et la construction des représentations sur les langues des répertoires des élèves. En effet, le Québec est marqué par des enjeux linguistiques prégnants autour du français (langue commune et officielle du Québec), de l'anglais (langue à renommée internationale et langue officielle du Canada) et des langues de l'immigration (Mc Andrew, 2010). Encore aujourd'hui, certaines représentations sont véhiculées autour de la nécessité de défendre le fait français face à la " menace » de l'anglais et des langues de l'immigration (Armand, 2013). Dans un tel contexte, la norme monolingue qui impose le français comme seule langue de l'école pour la très grande majorité des élèves immigrants et issus de l'immigration est prépondérante dans les milieux scolaires québécois, et les élèves en sont conscients.

Ainsi, les participants à notre recherche expriment une conscience aiguë de ces enjeux linguistiques environnants et l'intégration de la norme du français comme langue de l'école émerge, à des degrés divers, dans le discours de tous les participants. C'est dans cet esprit que nous supposons que, lors de la première entrevue, Laura n'a pas déclaré comme langue maternelle l'espagnol mais le français, que Shawn, devant ses enseignants, a tu durant tout le projet ses capacités à comprendre le créole et qu'Evan prend difficilement la parole devant le groupe-classe pour partager des connaissances en chinois, sa langue maternelle dans laquelle il suit des cours extrascolaires chaque samedi. Il est certain que d'autres facteurs jouent dans ces positionnements. Cependant, la norme monolingue imposée par l'école a indéniablement des effets sur les représentations des élèves quant aux langues de leur répertoire. Le contexte participe ainsi clairement à l'évolution des répertoires linguistiques des élèves, l'exemple éloquent d'Oliver dans sa biographie langagière nous permet d'illustrer ce phénomène :

Quand je suis à la maison, je me sens plus Haïtien et Seychellois que Québécois, parce que je peux parler d’autres langues que le français chez moi. (Oliver, module 6)

Alterstice-Revue Internationale de la Recherche Interculturelle, vol. 6, $n^{\circ} 1$ 
Précisons également que l'intégration de cette norme unique monolingue perdure et que, pour deux des élèves (Evan et Shawn), même après deux ans d'intervention d'éveil aux langues, elle représente encore une forme de barrière à l'apprivoisement de leur répertoire dans sa forme composite et plurielle. Pour les trois autres participants, cette conscience les amène à jouer de stratégies pour pouvoir, en fonction du contexte, aborder ou non, valoriser ou non, certaines de leurs langues. Dans le cadre de l'implantation du projet, il appert que ces élèves ont développé une flexibilité quant aux contextes, aux langues et, comme nous allons le voir dans la section suivante, aux interlocuteurs.

\section{Les interlocuteurs : les effets sur le caractère dynamique du répertoire linguistique des élèves}

L'analyse de nos données nous a également permis de constater que, en fonction des interlocuteurs, les élèves ajustent leurs comportements et leurs discours quant aux langues présentes dans leur répertoire. Dans cet esprit, et de toute évidence en lien avec les contextes d'interaction présentés plus haut, Shawn n'évoque jamais la présence du créole en groupe-classe. De même, et pour rappel, Evan ne partage pas volontairement ses connaissances en chinois avec ses camarades en petit groupe de travail et en groupe-classe. Également, Oliver adopte un positionnement similaire par rapport à ses langues d'origine (le créole seychellois et le créole haïtien) avec son enseignante Mathilde. Pour le module 3, les élèves sont invités à représenter sur un pétale de fleur les langues qu'ils parlent. L'enseignante (Mathilde) prend l'initiative d'ajouter dans la consigne que si les élèves ne sont pas en mesure de "décrire leur classe dans cette langue ", ils ne peuvent pas la considérer comme langue parlée. Le choix discutable de l'enseignante n'est pas débattu dans le cadre de cette analyse, mais il nous permet d'éclairer les positionnements d'Oliver quant à ses langues d'origine lorsque son enseignante est présente. Ainsi, dans les activités de classe qui engagent un partage entre camarades, Oliver mentionne la présence de ses deux créoles au sein de son répertoire. Toutefois, dans la présentation de son pétale de fleur devant le groupe classe qui inclut son enseignante, il exprime la présence d'un seul créole qu'il ne spécifie d'ailleurs pas afin de rester assez vague pour ne pas remettre en jeu ses déclarations et son désir d'affirmer le créole comme langue de son répertoire. À l'inverse, aux entrevues 2 et 3, durant lesquelles les enseignants sont absents, Oliver ne cache pas la présence des deux créoles au sein de son répertoire.

Dans un même ordre d'idées, Laura aborde son bilinguisme espagnol-français de façon différente selon qu'elle le situe avec ses parents ou à l'extérieur de la cellule familiale. Par exemple, elle explique lors d'un échange informel avec le caméraman au module 6 qu'elle " mélange » fréquemment le français et l'espagnol et que cette pratique fait rire ses parents. Dans le discours de l'élève, ce rire semble vouloir marquer une forme de complicité familiale qui, finalement, participerait à la normalisation et à l'acceptation d'un parler bilingue français-espagnol. Toutefois, un second "rire", celui des "gens » qui sont hors du noyau familial, serait quant à lui connoté négativement et porteur de jugements sur ses compétences linguistiques puisqu'elle indique :

Comme il y a des gens qui sont, on va dire, qu'ils parlent juste comme espagnol. Puis après, je parle en espagnol, mais j'ai peur de mélanger les mots et puis que les gens rient de moi. (Laura, module 6)

Baker (2011), Cummins (2001, 2014) et Hélot (2007) ont souligné le poids du discours des enseignants dans la construction des représentations sur les langues des élèves. Dans cette même lignée, nos données soulignent, en contexte scolaire, une certaine sensibilité des apprenants quant aux discours de " modèles " adultes qui peuvent les entourer. Dans notre recherche, ces modèles prennent la forme des enseignants, mais aussi des chercheures impliquées dans l'implantation du projet Élodil. Les élèves font ainsi preuve de leur lucidité quant à la possibilité d'intégrer ou non des langues au sein de leur répertoire en fonction des interactants en présence : ils choisissent soit de les passer sous silence, soit, au contraire, de les valoriser.

\section{Conclusion}

Les résultats de recherche présentés dans cet article permettent de documenter de façon qualitative et sur une période de deux ans d'implantation d'un projet d'éveil aux langues les nuances que le temps, le contexte et les interlocuteurs peuvent apporter dans la dynamisation des répertoires plurilingues de cinq élèves. Nous avons ainsi pu voir que, même si les représentations sur les langues exprimées par les élèves peuvent être marquées par le poids des représentations sociales, cette tendance s'atténue au fur et à mesure de l'implantation du projet et une 
majorité d'élèves exprime des représentations plus individualisées en fin de projet. De plus, pour la majorité d'entre eux, le sentiment d'insécurité linguistique partagé autour de l'une ou l'autre de leurs langues s'estompe au fil du temps et ils mettent en place des stratégies qui leur permettent de mieux apprivoiser leur répertoire linguistique dans leur forme composite. Les élèves font également la preuve de leur capacité à s'ajuster aux situations de communication en fonction des interlocuteurs et des contextes, en mettant par exemple de l'avant ou non certaines langues de leur répertoire.

L'implantation des activités Élodil a donc eu des effets favorables sur les représentations à propos des langues des répertoires linguistiques des élèves participant à la recherche. Dans un contexte tel que celui du Québec, où le système scolaire est trop souvent frileux à la prise en compte de la diversité linguistique, l'éveil aux langues semble

donc être une approche intéressante pour favoriser la mise en place d'un espace de "complicité linguistique ", où les langues des élèves, les langues de l'école et les langues du monde deviennent un objet de partage, de savoir, mais aussi de fierté.

\section{Références bibliographiques}

Armand, F. (2012). Enseigner en milieu pluriethnique et plurilingue : place aux pratiques innovantes. Québec français, 167, 48-50.

Armand, F. (2013). Former les futurs enseignants à œuvrer en contextes de diversité : une priorité au Québec. Québec français, 168, 83-85.

Armand, F., Dagenais, D. et Nicollin, L. (2008). La dimension linguistique des enjeux interculturels : de l'éveil aux langues à l'éducation plurilingue. Éducation et francophonie, 36(1), 44-64.

Baker, C. (2011). Foundations of bilingual education and bilingualism. Clevedon : Multilingual Matters.

Blommaert, J., Collins, J. et Slembrouck, S. (2005). Spaces of multilingualism. Language and Communication, 25(3), 197-206.

Bono, M. et Stratilaki, S. (2009). The M-factor, a bilingual asset for plurilinguals ? Learners' representations, discourse strategies and third language acquisition in institutional contexts. International Journal of Multilingualism, 6(2), 207-227.

Candelier, M. (2006). Éveil aux langues, formation plurilingue et enseignement du français. Synergie Monde, 1, 6776.

Candelier, M. (2008). Approches plurielles, didactiques du plurilinguisme : le même et l'autre. Les Cahiers de l'Acedle, 5(1), 65-90.

Castellotti, V. et Moore, D. (2002). Représentations sociales des langues et enseignement, Guide pour l'élaboration des politiques linguistiques éducatives en Europe - De la diversité linguistique à l'éducation plurilingue.

Strasbourg : Conseil de l'Europe, Division des politiques linguistiques.

Cummins, J. (2001). Negotiating identities: Education for empowerment in a diverse society. Los Angeles : California Association for Bilingual Education.

Cummins, J. (2007). Language Power and Pedagogy - Bilingual Children in the Crossfire. Édition revue et corrigée 2007. Clevedon : Multilingual Matters.

Cummins, J. (2014). L'éducation bilingue : qu'avons-nous appris de cinquante ans de recherche ? Dans I. Nocus, J. Vernaudon et M. Paia (dir.), L'école plurilingue en Outre-mer: Apprendre plusieurs langues, plusieurs langues pour apprendre (p. 41-63). Rennes: Presses universitaires de Rennes.

Cummins, J. et Early, M. (2011). Identity texts: The collaborative creation of power in multilingual schools. Stoke-onTrent, England : Trentham Books.

Deprez, C. (1994). Les enfants bilingues : langues et familles. Paris : Didier-Crédif. 
Dompmartin-Normand, C. (2003). Éveil et ouverture aux langues à l'école : contribution à l'évaluation d'un outil en région toulousaine (Thèse de doctorat inédite). Université de Toulouse.

Dörnyei, Z. (2007). Research Methods in Applied Linguistics. Quantitative, Qualitative and Mixed Methodologies. Oxford : Oxford University Press.

Élodil (2010). Éveil au langage et ouverture à la diversité linguistique [en ligne]. www.elodil.com (premier site) et http://www.elodil.umontreal.ca (ensemble des activités)

Garcia, O. (2009). Education, Multingualism and Translanguaging in the $21^{\text {st }}$ century. Dans A.K. Mohanty, M. Panda, R. Phillipson et Skunabb-Kangas (dir.), Multilingual Education for Social Justice : Globalising the Local. New Delhi : Orient BlackSwan.

Garcia, O. et Kleifgen, J. (2010). Emergent Bilinguals, Policies, Programs, and Practices for English Language Learners. New York : Columbia University.

Genesee, F., Paradis, J. et Crago, B. (2004). Dual Language Development \& Disorders. A Handbook on Bilingualism and Second Language Learning. Baltimore : Brookes Publishing.

Grosjean, F. (1982). Life with two languages. Cambridge, MA : Harvard University Press.

Hamers, J. (2005). Le rôle de la L1 dans les acquisitions ultérieures. Dans L.F. Prudent, F. Tupin et S. Wharton (dir.), Du plurilinguisme à l'école (p. 271-292). Berne : Peter Lang.

Hélot, C. (2007). Du bilinguisme en famille au plurilinguisme à l'école. Paris : L'Harmattan.

Hélot, C. et Ó Laoire, M. (2011). Language Policy for the Multilingual Classroom: Pedagogy of the Possible. Clevedon : Multilingual Matters.

Jodelet, D. (1989). Représentations sociales : un domaine en expansion. Dans D. Jodelet (dir.), Les représentations sociales (p. 47-78). Paris : Presses universitaires de France.

Krippendorff, K. (2013). Content analysis: An introduction to its methodology. Sage Publications.

Lory, M.-P. (2015). L'évolution sur les langues d'élèves plurilingues de $3^{e}$ cycle du primaire lors de l'implantation d'un projet d'Éveil aux langues (Thèse de doctorat, Université de Montréal). https://papyrus.bib.umontreal.ca/xmlui/handle/1866/13048.

Lüdi, G. et Py, B. (2002). Être bilingue (2 éd.). Berlin : Peter Lang.

Lüdi, G., et Py, B. (2009). To be or not to be... a plurilingual speaker. International Journal of Multilingualism, 6(2), 154-167.

Maingueneau, D. (2007). Analyser les textes de communication. Paris : Armand Colin.

Mc Andrew, M. (2010). Les Majorités fragiles de l'éducation. Québec : Presses de l'Université de Montréal.

Moore, D. (2006). Plurilinguisme et école. Paris : Didier.

Moore, D. et Castellotti, V. (2008). La compétence plurilingue : regards francophones. Berne : Peter Lang.

Moseley, Christopher (dir.). (2010). Atlas des langues en danger dans le monde ( ${ }^{\mathrm{e}}$ éd). Paris : Éditions Unesco. Version en ligne : http://www.unesco.org/culture/en/endangeredlanguages/atlas

Norton, B. et Toohey, K. (2011). Identity, language learning and social change. Language teaching, 44, 412-446.

Paillé, P. (1996). De l'analyse qualitative en général et de l'analyse thématique en particulier. Revue de l'Association pour la recherche qualitative, 15, 179-84.

Prasad, G. (2015). The Prism of Children's Plurilingualism: A multi-site inquiry with children as co-researchers across English and French schools (Thèse de doctorat, Université de Toronto).

Puezzo Capron, I. et Picardo, E. (2013). L'émotion et l'apprentissage des langues. Revue de linguistique et de didactique des langues, 48. 
Stake, R. (2006). Multiple Case Study Analysis. New York : Guilford Press.

Statistique Canada (2011). Immigration et diversité culturelle au Canada [en ligne]. Ottawa : Statistique Canada. https://www12.statcan.gc.ca/nhs-enm/2011/as-sa/99-010-x/99-010-x2011001-fra.cfm

Unesco. (2003). Vitalité et disparition des langues. Paris : Unesco. http://www.unesco.org/culture/ich/doc/src/00120-FR.pdf 